\title{
ЭЛЕКТРОМАГНИТНАЯ СУШКА ВЛАЖНЫХ МАТЕРИАЛОВ С МАЛОЙ ГЛУБИНОЙ ПРОНИКНОВЕНИЯ СВЧ-ИЗЛУЧЕНИЯ В УСЛОВИЯХ ТЕПЛОСБРОСА РАДИАЦИЕЙ И КОНВЕКЦИЕЙ. І. СТАДИЯ ПРОГРЕВА
}

Саломатов Владимир Васильевич ${ }^{1,2}$, salomatov.vv@mail.ru

\section{Карелин Вадим Александрович,2,} vad2hen@mail.ru

\author{
Саломатов Василий Владимирович², \\ vvsalomatov@mail.ru \\ 1 Новосибирский государственный университет, \\ Россия, 630090, г. Новосибирск, ул. Пирогова, 1. \\ 2 Институт теплофизики им. С.С. Кутателадзе СО РАН, \\ Россия, 630090, г. Новосибирск, пр. Академика Лаврентьева, 1.
}

\begin{abstract}
Актуальность исследования продиктована необходимостью разработки математических моделей СВЧ-нагрева и МВ-сушки влажных материалов для получения технологически оптимальных и экономически выгодных режимов. В силу сложности и нелинейности протекающих процессов внутри влажных материалов при СВЧ-обработке чрезвычайно важно построение моделей, которые давали бы возможность построения аналитически приближенных решений с целью нахождения на их основе главных закономерностей и характерных особенностей рассматриваемых процессов.

Цель: постановка первого этапа задачи СВЧ-сушки влажного материала - этапа прогрева. Поиск аналитического решения для температуры слоя в разные моменты времени позволяет определить начало следующего этапа - сушки.

Объектом исследования является плоский слой влажного материала - уголь, песок, древесина и др. капиллярно-пористые массивы, на которые воздействует СВЧ-излучение. Такие материалы обладают высокой диэлектрической проницаемостью и как следствие весьма эффективно поглощают СВЧ-излучение, которое почти на 100 \% преобразуется в тепловую энергию.

Методы исследования связаны с математическим моделированием, в основе которого лежат уравнения электродинамики Максвелла и тепловлагопереноса А.В. Лыкова. В данной статье задача Максвелла решена независимо от задачи тепломассопереноса, поэтому плотность потока поглощенного СВЧ-излучения считается известной. Также одной из особенностей данной задачи является рассмотрение материалов с малой глубиной поглощения, в силу чего в системе уравнений для нагрева источниковый член находится в граничном условии.

В результате исследования с помощью асимптотических процедур решена задача тепловлагопереноса при заданной мощности источника тепла. Получены приближенно-аналитические решения для первой из трех характерных стадий СВЧ-сушки в условиях малой глубины проникновения микроволн: стадии прогрева влажного материала. Выполнен анализ построенных решений.
\end{abstract}

\section{Ключевые слова:}

СВЧ-энергия, сушка, капиллярно-пористый массив, теплоизлучение, конвекция, уравнения тепловлагопереноса А.В. Лыкова.

\section{Введение}

Сушка влажных материалов является важным технологическим процессом в ряде отраслей промышленности, таких как: производство древесины, строительная индустрия, химия и др., а также в сельском хозяйстве. Масштабы использования сушки особенно значительны в угольной энергетике, так как на тепловые электрические станции и котельные, как правило, поступает влажное топливо, которое для сжигания необходимо подсушивать (по российским требованиям до конечной влажности в 10 \%) [1-4]. Дальнейшее повышение эффективности угольных ТЭС и выполнение ужесточенных экологических норм заставляет разрабатывать новые технологии термообработки энергетических углей. Большой потенциал имеют способы обработки угля с участием СВЧ-энергии [5-9]. Важнейшим преимуществом СВЧ-потока является способность электромагнитного излучения проникать в подсушиваемый материал на значительную глуби- ну, создавая в нем объемное распределение внутренних источников тепла. СВЧ-поле является регулируемым и практически безынерционным процессом, что дает возможность мгновенно дозировать подачу тепла. Так, одной из главных особенностей СВЧ-энергии является её избирательный характер, поэтому отдельные составляющие многокомпонентного материала нагреваются по-разному, в зависимости от их диэлектрических свойств. Эта особенность широко используется при СВЧсушке, когда вода испаряется из материала, при этом сам материал сохраняет свои качества. В условиях СВЧ-сушки обнаружено, что при испарении влаги из угля одновременно происходит удаление азота и снижение серы этих углей [10]. Особо важно отметить, что энергозатраты на испарение при традиционных видах сушки составляют до 3,0 кВт॰ч/кг, а при СВЧ-сушке они снижаются до $1,6-1,8$ кВт•ч/кг. Время традиционной сушки находится в пределах 8-20 часов, а в условиях СВЧ- 
сушки при прочих равных условиях оно уменьшается до 4 часов [11]. Кроме улучшения экологических и экономических факторов применение СВЧэнергии в процессе сушки угольного топлива повышает взрывобезопасность, снижает металлоёмкость сушильных агрегатов. Таким образом, использование микроволнового излучения для сушки угля отвечает задачам инновационного развития твердотопливной энергетики [12-15].

Дальнейшее изучение проблемы СВЧ-сушки требует разработки математических подходов с целью получения расчетных данных, с помощью которых обеспечивается экологичность, экономичность, энергоэффективность, безопасность и другие качественные стороны технологии СВЧ-сушки. Широкому тиражированию СВЧ-технологий в угольной энергетике, к которым в мире на сегодня проявляется повышенный интерес, мешает главным образом слабая разработанность научных основ взаимосвязанных, достаточно сложных электрофизических и теплофизических процессов. Использовать в полной мере преимущества микроволнового воздействия на качественную термоподготовку угля и его сушку можно лишь при наличии: во-первых, современных математических моделей и средств их реализации; во-вторых, банка экспериментальных данных воздействия СВЧ на влажный уголь. Как указывают результаты анализов патентов и опубликованных статей, использование подверженных СВЧ-излучению низкосортных углей обеспечивает улучшение работы угольных ТЭС в следующих направлениях: повышение общей эффективности, снижение эксплуатационных затрат, расширение возможностей одновременного использования разных марок углей, оздоровление окружающей среды [16-18].

\section{Математическая модель электромагнитной сушки влажного плоского слоя}

Примем следующие допущения:

- образец сушки - неограниченная пластина толщиной $l(\mathrm{M})$;

- из-за малой глубины проникновения СВЧ-излучения подводимая к поверхности $x=l$ (рис. 1) плотность теплового потока $q_{w}$ выражается следующим образом

$$
q_{w}=A W .
$$

- теплофизические и электрофизические характеристики материала - постоянные величины;

- теплоотвод поглощенной СВЧ-энергии осуществляется конвекцией и радиацией одновременно.

C учетом принятых допущений математическая модель представляется в виде следующей системы дифференциальных уравнений:

$$
\begin{gathered}
c \rho_{0} \frac{\partial T}{\partial t}=\lambda \frac{\partial^{2} T}{\partial x^{2}}+r \varepsilon \rho_{0} \frac{\partial u}{\partial t}, \\
\frac{\partial u}{\partial t}=a_{m} \frac{\partial^{2} u}{\partial x^{2}}+a_{M}^{T} \frac{\partial^{2} T}{\partial x^{2}},
\end{gathered}
$$

$$
\begin{gathered}
\frac{\partial T(0, t)}{\partial x}=0, \frac{\partial u(0, t)}{\partial x}=0, \\
T(x, 0)=T_{0}, u(x, 0)=u_{0}, \\
\lambda \frac{\partial T(l, t)}{\partial x}=q_{w}-\alpha\left[T(l, t)-T_{c}\right]- \\
-\varepsilon_{\mathrm{q}} \sigma_{0}\left[T^{4}(l, t)-T_{c}^{4}\right]+r(1-\varepsilon) j(l, t),
\end{gathered}
$$
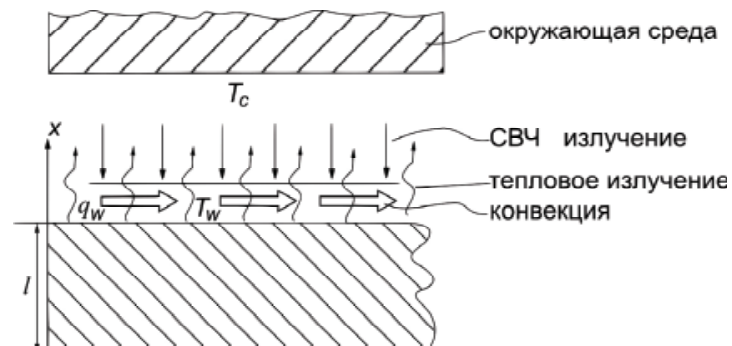

Puс. 1. Схема электромагнитной сушки влажного плоского массива в условиях малой глубины проникновения СВЧ-излучения

Fig. 1. Scheme of electromagnetic drying of a wet flat massif under conditions of low penetration depth of microwave radiation

Используя масштабы задачи, входящие в условия однозначности, переведем систему 1.1-1.6 к безразмерному виду $1.7-1.14$. В результате получим

$$
\begin{aligned}
& \frac{\partial \Theta(X, \mathrm{Fo})}{d \mathrm{Fo}}=(1+\varepsilon K o P n L u) \frac{\partial^{2} \Theta(X, \mathrm{Fo})}{\partial X^{2}}+ \\
& +\varepsilon K o L u \frac{\partial U(X, \mathrm{Fo})}{\partial X}, \\
& \frac{\partial U(X, \mathrm{Fo})}{\partial \mathrm{Fo}}= \\
& =L u \frac{\partial^{2} U(X, \mathrm{Fo})}{\partial X^{2}}-L u P n \frac{\partial^{2} \Theta(X, \mathrm{Fo})}{\partial X^{2}}, \\
& \frac{\partial \Theta(1, \mathrm{Fo})}{\partial \mathrm{Fo}}=K i_{q}-(1-\varepsilon) L u K o K i_{m}(\mathrm{Fo})=0, \\
& \frac{\partial U(1, \mathrm{Fo})}{\partial X}=P n \frac{\partial \Theta(1, \mathrm{Fo})}{\partial X}+K i_{m}(\mathrm{Fo})=0, \\
& \frac{\partial \Theta(0, \mathrm{Fo})}{\partial X}=0, \\
& \frac{\partial U(0, \mathrm{Fo})}{\partial X}=0, \\
& \Theta(X, 0)=\Theta_{0}, \\
& U(X, 0)=U_{0} .
\end{aligned}
$$

Задачу прогрева влажного массива (в условиях малой глубины проникновения СВЧ-излучения) можно представить в виде 1.15-1.18 


$$
\begin{aligned}
& \frac{\partial T(x, t)}{\partial t}=\grave{a} \frac{\partial^{2} T(x, t)}{\partial x^{2}}, \\
& T(x, 0)=T_{0}, \\
& -\lambda \frac{\partial T(l, t)}{\partial x}= \\
& =q_{w}-\left[\alpha\left(T_{w}-T_{c}\right)+\varepsilon_{\dot{亠}} \sigma_{0}\left(T_{w}^{4}-T_{c}^{4}\right)\right], \\
& \frac{\partial T(0, t)}{\partial x}=0 .
\end{aligned}
$$

Перейдем к безразмерным переменным

$$
\begin{gathered}
X=\frac{x}{l}, \quad F O=\frac{a t}{l^{2}}, K i=\frac{A W l}{\lambda T_{c}}, \\
\Theta=\frac{T}{T_{c}}, S k=\frac{\varepsilon_{\mathrm{q}} \sigma_{0} T_{c}^{3} l}{\lambda}, B i=\frac{\alpha l}{\lambda} .
\end{gathered}
$$

В результате имеем

$$
\begin{gathered}
\frac{\partial \Theta}{\partial \mathrm{Fo}}=\frac{\partial^{2} \Theta}{\partial X^{2}}, \\
\Theta(X, 0)=\Theta_{0}, \\
\frac{\partial \Theta(1, \mathrm{Fo})}{\partial X}= \\
=K i_{w}-\left[B i\left(\Theta_{w}-1\right)+S k\left(\Theta_{w}^{4}-1\right)\right] \equiv Q(\mathrm{Fo}), \\
\frac{\partial \Theta(0, \mathrm{Fo})}{\partial X}=0 .
\end{gathered}
$$

Переходим в $1.19-1.22$ к преобразованной системе по Лапласу

$$
\begin{gathered}
s \Theta_{L}(X, s)-\Theta_{0}=\Theta_{L}^{\prime \prime}(X, s), \\
\Theta_{L}^{\prime}(1, s)=Q_{L}(s), \\
\Theta_{L}^{\prime}(0, s)=0 .
\end{gathered}
$$

Общее решение $1.23-1.25$ для изображения представляется в виде:

$$
\Theta_{L}(X, s)-\frac{\Theta_{0}}{s} \approx Q_{L}(s) F(X, s),
$$

где $F(X, s)$ - передаточная функция.

Далее в силу нелинейности сформулированной задачи перейдём к построению ее асимптотических решений. Используется следующее свойство преобразования Лапласа: малым числам Фурье соответствуют большие значения параметра преобразования $s$, и наоборот, большим числам Фурье малые значения этого параметра.

\section{Асимптотика при малых Fo (большие s)}

Разложим передаточную функцию из 1.26 в ряд по большому параметру:

$$
F(X, s) \approx \varphi_{1}(X, s) \frac{e^{-\sqrt{s}}}{\sqrt{s}}+\varphi_{2}(X, s) \frac{e^{-s}}{s}+\ldots
$$

Подставляя 1.27 в 1.26 и удовлетворяя системе уравнения $1.23-1.25$, получим следующую цепочку уравнений $1.28,1.29$ для нахождения коэффициентов разложения $\varphi_{1}(X, s), \varphi_{2}(X, s), \varphi_{3}(X, s)$

$$
\begin{gathered}
\varphi_{1}^{\prime \prime}(X, s)-s \varphi_{1}(X, s)=0 ; \\
\varphi_{1}^{\prime}(1, s)=\frac{\sqrt{s}}{e^{-\sqrt{s}}} ; \\
\varphi_{1}^{\prime}(0, s)=0, \\
\varphi_{2}^{\prime \prime}(X, s)-s \varphi_{2}(X, s)=0 ; \\
\varphi_{2}^{\prime}(1, s)=0 ; \\
\varphi_{2}^{\prime}(0, s)=0 .
\end{gathered}
$$

В итоге разложение 1.27 при больших $\mathrm{s}$ приобретает вид

$$
\begin{gathered}
\Theta_{L}(X, s)-\frac{\Theta_{0}}{s} \approx \\
\approx \frac{Q_{L}(s)}{\sqrt{s}}\left[e^{-\sqrt{s}(X+1)}+e^{-\sqrt{s}(1-X)}\right]+\ldots
\end{gathered}
$$

Возвращаясь в пространство оригиналов, получим решение при малых Fo.

Оригинал выражения $\frac{\left[e^{-\sqrt{s}(x+1)}+e^{\sqrt{s}(X-1)}\right]}{\sqrt{s}}$ можно записать в виде 1.31:

$$
\frac{e^{-\frac{(1-X)^{2}}{4 F o}}+e^{-\frac{(X+1)^{2}}{4 F o}}}{\sqrt{\pi F O}} .
$$

Так как $Q$ в 1.30 содержит нелинейность из-за наличия закона Стефана-Больцмана для получения расчётного соотношения, разложим $Q(\eta)$ вблизи $\eta \approx$ Fо в ряд Тейлора:

$$
Q(\eta) \approx Q(F o)+(\eta-F o) Q^{\prime}(F o)+\ldots
$$

Ограничиваясь при малых Fо первым членом разложения, находим свертку 1.32:

$$
\begin{aligned}
& \int_{0}^{F_{o}} Q(\eta) \frac{e^{-\frac{(X-1)^{2}}{4(F o-\eta)}}+e^{-\frac{(X+1)^{2}}{4(F o-\eta)}}}{\sqrt{\pi(F o-\eta)}} d \eta=
\end{aligned}
$$

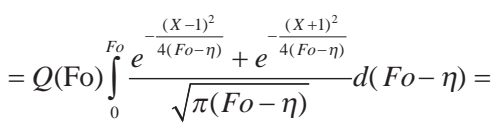

$$
\begin{aligned}
& =Q(F o) \int_{0}^{F_{0}} \frac{e^{-\frac{(X-1)^{2}}{4 y}}+e^{-\frac{(X+1)^{2}}{4 y}}}{\sqrt{\pi y}} d y=
\end{aligned}
$$

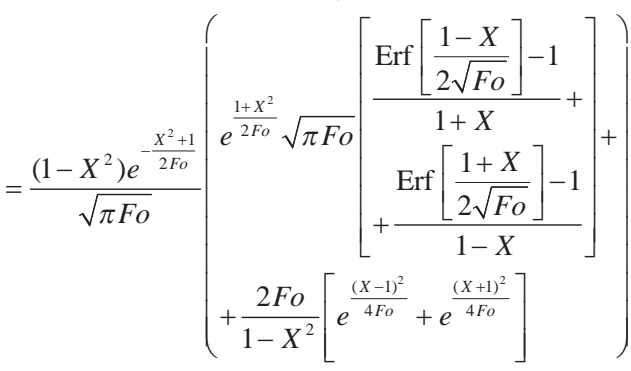

и в итоге решение выглядит следующим образом: 
$\Theta(X, F o) \approx \Theta_{0}+\left[K i-B i\left(\Theta_{w}-1\right)-S k\left(\Theta_{w}{ }^{4}-1\right)\right]\left(1-X^{2}\right)$

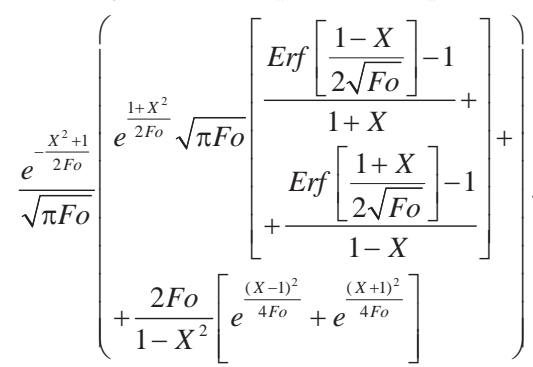

При $X=1$ : имеем уравнение для температуры поверхности

$$
\begin{gathered}
\Theta(1, F O) \approx \Theta_{0}+\left[K i-B i\left(\Theta_{w}-1\right)-S k\left(\Theta_{w}{ }^{4}-1\right)\right] \times \\
\times 2 \sqrt{\frac{F O}{\pi}}\left(e^{-\frac{1}{F o}}-\sqrt{\frac{\pi}{F O}} \operatorname{Erfc}\left[\frac{1}{\sqrt{F O}}\right]+1\right) .
\end{gathered}
$$

Для записи итоговой граничной температуры введём несколько обозначений:

$$
\begin{aligned}
& A(F O)=\sqrt{\frac{\pi}{F O}} e^{\frac{1}{F o}} \operatorname{ErfC}\left[\frac{1}{\sqrt{F_{O}}}\right]-1-e^{\frac{1}{F_{0}}} ; \\
& B(F o, B i, S k)=27\left(\frac{B i}{S k \cdot A}\right)^{2} \times \\
& \times\left(\begin{array}{c}
\left.1+2 e^{\frac{1}{F O}}+e^{\frac{2}{F o}}-\left(\sqrt{\frac{\pi}{F O}} \operatorname{Erfc}\left[\frac{1}{\sqrt{F O}}\right]\right) \times\right) \\
\times\left(2 e^{\frac{1}{F O}}+2 e^{\frac{2}{F o}}\right)+\frac{e^{\frac{2}{F O}}}{F O} \pi \operatorname{Erfc}^{2}\left[\frac{1}{\sqrt{F O}}\right]
\end{array}\right)+
\end{aligned}
$$

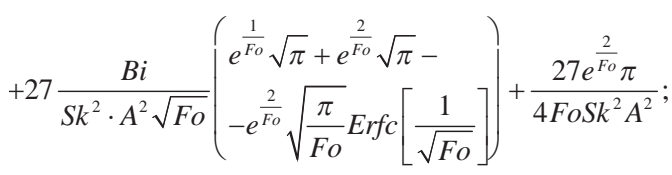

$$
\begin{aligned}
& C(\mathrm{Fo}, \mathrm{Bi}, \mathrm{Sk}, \mathrm{Ki})=\frac{864}{\mathrm{Fo}^{\frac{3}{2}} S k^{3} A^{3}} \times \\
& \times\left(\begin{array}{l}
-2 \sqrt{F O}\left[1+e^{\frac{1}{F o}}-e^{\frac{1}{F o}} \sqrt{\frac{\pi}{F O}} \operatorname{ErfC}\left[\frac{1}{\sqrt{F O}}\right]\right] \times \\
\times[B i+K i+S k]-e^{\frac{1}{F o}} \sqrt{\pi} \theta_{0}
\end{array}\right)^{3} ; \\
& D(F o, B i, S k, K i)=\frac{2^{\frac{1}{3}}}{A\left(B+\sqrt{C+B^{2}}\right)^{\frac{1}{3}}} .
\end{aligned}
$$

Введем также дополнительную функцию:

$$
\begin{gathered}
Z(F o, S k, B i, K i)= \\
=-4 D A+\frac{4 D}{S k}\left(-(B i+K i) A+\frac{\sqrt{\pi} \theta_{0} e^{\frac{1}{F o}}}{2 \sqrt{F_{O}}}\right)+\frac{1}{3 D A},
\end{gathered}
$$

через которую можно довольно просто записать окончательное выражение:

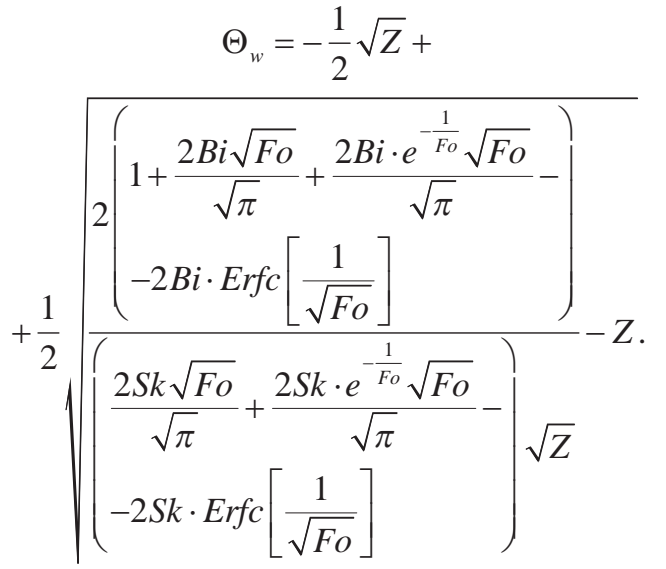

В итоге, зная граничную температуру 1.34 , становится возможным определить температуру по глубине слоя с течением времени:

$$
\begin{gathered}
\Theta(X, F o) \approx \Theta_{0}+\left[K i-B i\left(\Theta_{w}-1\right)-S k\left(\Theta_{w}{ }^{4}-1\right)\right]\left(1-X^{2}\right) . \\
\frac{e^{-\frac{X^{2}+1}{2 F o}}}{\sqrt{\pi F O}}\left(\begin{array}{l}
e^{\frac{1+X^{2}}{2 F o}} \sqrt{\pi F o}\left[\frac{E r f\left[\frac{1-X}{2 \sqrt{F O}}\right]-1}{1+X}+\frac{\operatorname{Erf}\left[\frac{1+X}{2 \sqrt{F O}}\right]-1}{1-X}\right]+ \\
+\frac{2 F O}{1-X^{2}}\left[e^{\frac{(X-1)^{2}}{4 F o}}+e^{\frac{(X+1)^{2}}{4 F o}}\right]
\end{array}\right) .
\end{gathered}
$$

Для проверки найденного решения рассмотрим случай с нулевым сбросом тепла: $B i=S k=0$. В этом примере поверхностная температура будет иметь вид:

$$
\Theta_{w} \approx \Theta_{0}+2 K i \sqrt{\frac{F O}{\pi}}\left(e^{-\frac{1}{F o}}-\sqrt{\frac{\pi}{F O}} \operatorname{Erfc}\left[\frac{1}{\sqrt{F_{O}}}\right]+1\right) .
$$

А для тестового примера $K i=9$ и начальной температуры, равной 1 , можем определить вид зависимости безразмерной поверхностной температуры от числа $F o$, представленный нарис. 2.

Распределение температуры по глубине при $\mathrm{F}_{0}=0,1$ имеет вид как на рис. 3 .

Приведем также аналогичные графики для случая с теплоотводом $(\mathrm{Bi}=5, \mathrm{Ki}=9, \mathrm{Sk}=0,5)$ (рис. 4, 5).

Далее сравним полученное решение с решением одной из известных задач А.В. Лыкова [19]. Данные решения сравнивались при равных соответствующих параметрах. Сравнение приведено ниже (рис. 6, 7).

Процентная разность безразмерных температур при различных числах Fo.

Видно, что при $\mathrm{Fo}_{0}<0,5$ разность температур для расчетной задачи А.В. Лыкова и полученного решения 1.33 составляет не более 1,7 \%.

Также приведем график двух температур (рис. 7). 


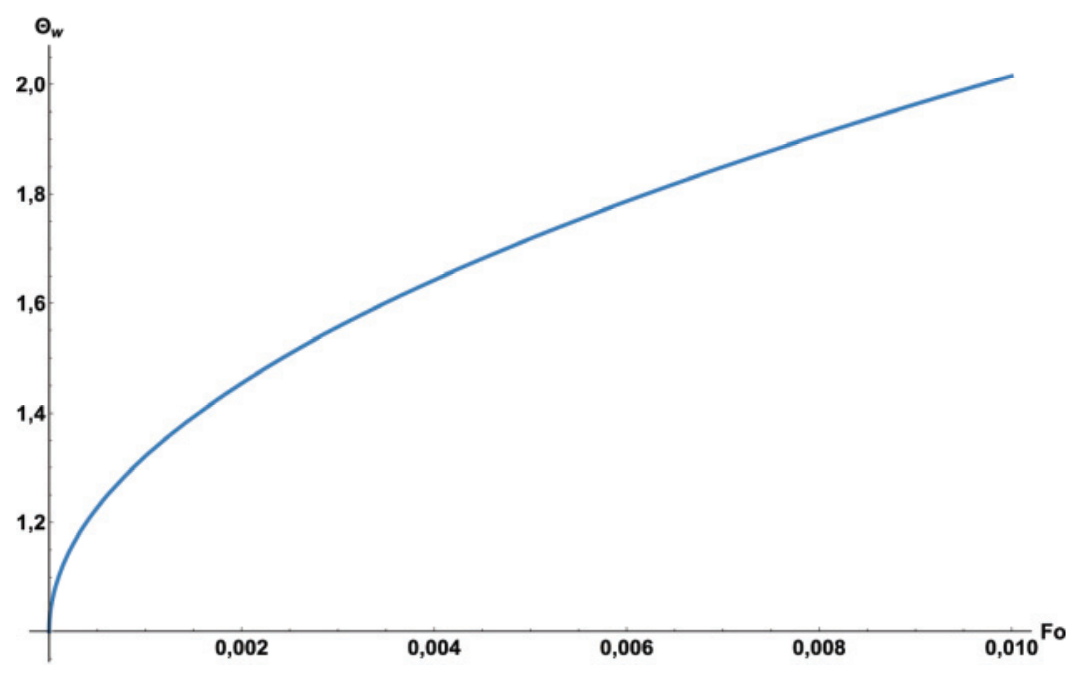

Рис. 2. Зависимость граничной температуры от числа Фурье в отсутствии теплоотвода

Fig. 2. Dependence of the boundary temperature on the Fourier number without heat removal

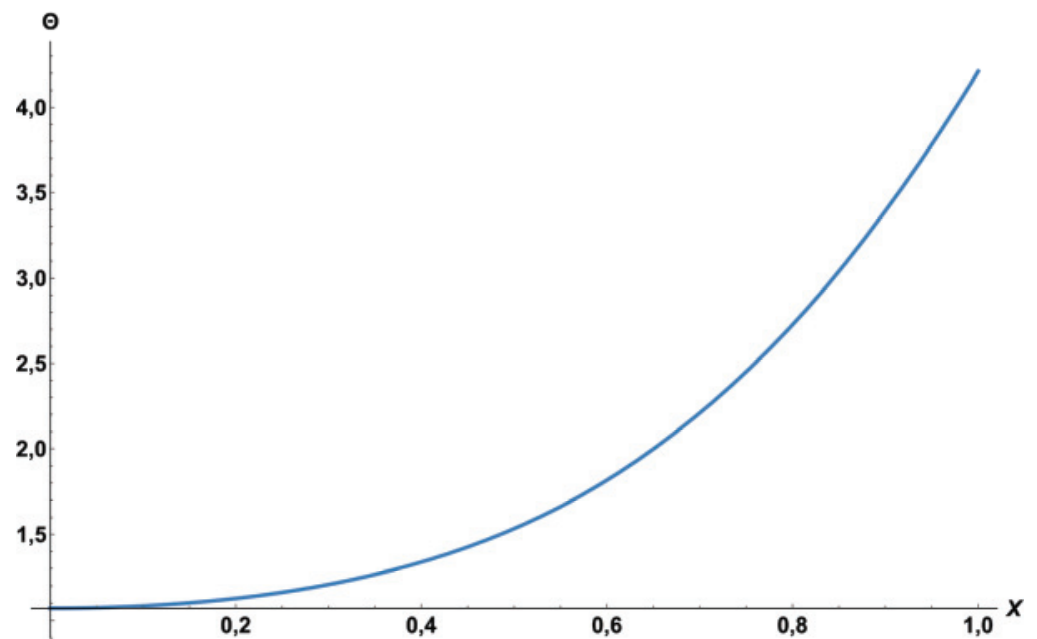

Pис.3. Зависимость температуры от координаты слоя ( $x=1$ - гранииа, на которую падает СВЧ-волна) в момент времени $F o=0,1$ в отсутствии теплоотвода

Fig. 3. Dependence of temperature on the layer coordinate ( $x=1$ - the boundary on which the microwave falls) at the instant of time $F_{0}=0,1$ without heat removal

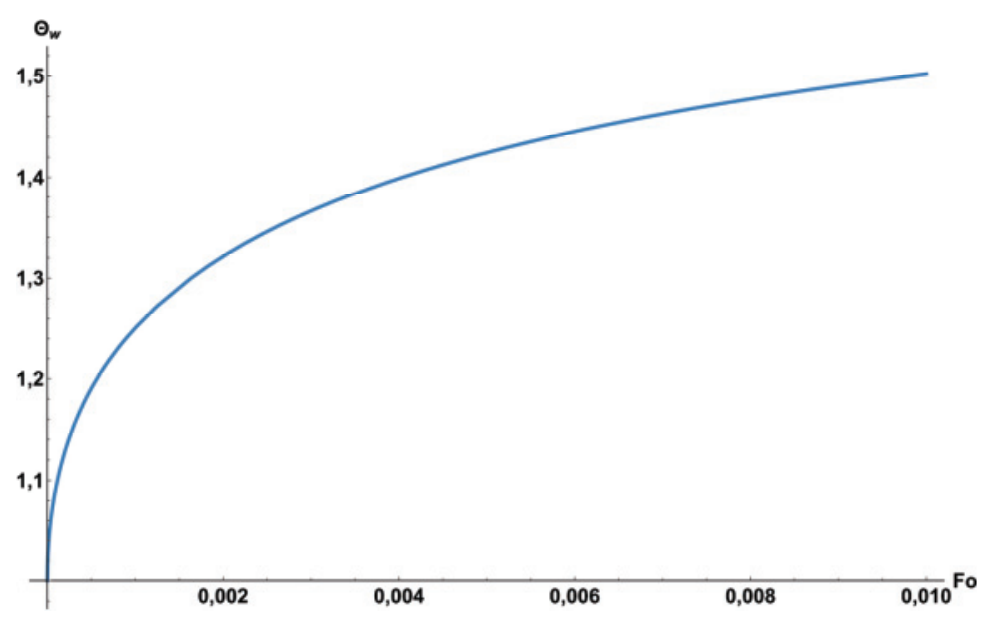

Рис. 4. Зависимость граничной температуры от числа Фурье с теплоотводом

Fig. 4. Dependence of the boundary temperature on the Fourier number with heat removal 


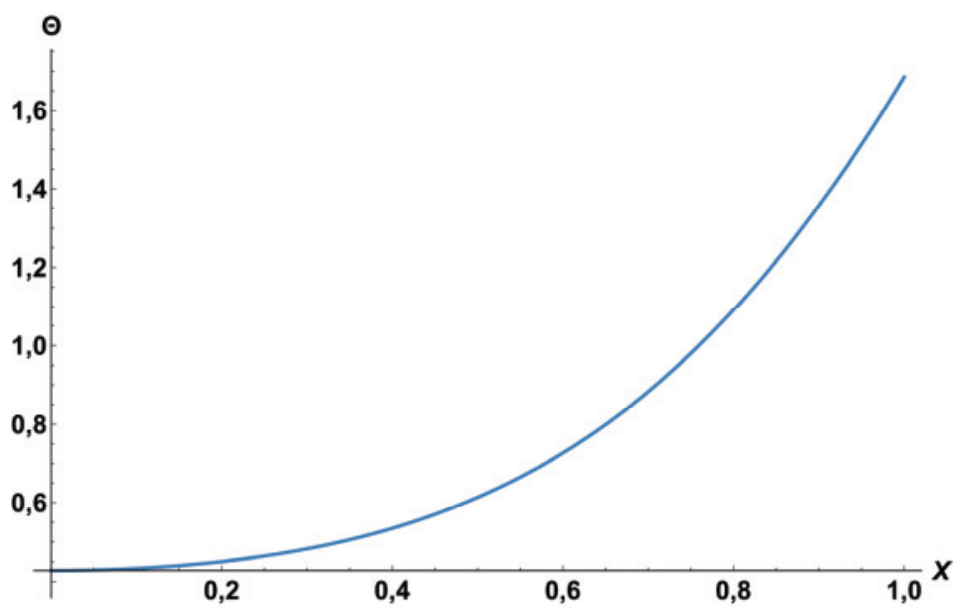

Puс. 5. Зависимость температуры от координаты слоя (X=1 - гранииа, на которую падает СВЧ-волна) в момент врелени Fо=0,1 с теплоотводол

Fig. 5. Dependence of temperature on the layer coordinate $(X=1-$ boundary, on which the microwave wave falls $)$ at time Fo=0,1 with heat removal

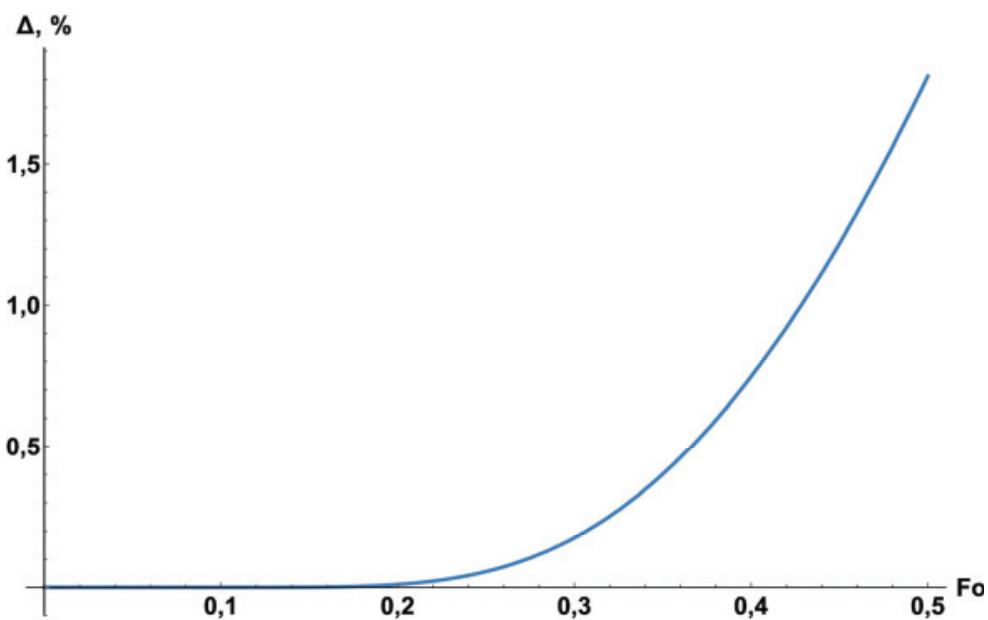

Pис. 6. Процентное расхождение точного решения задачи Лыкова [19] и асимптотического решения, полученного для мальх чисел Fо

Fig. 6. Percentage divergence of the exact solution of the Lykov [19] problem and the asymptotic solution obtained for small numbers Fo

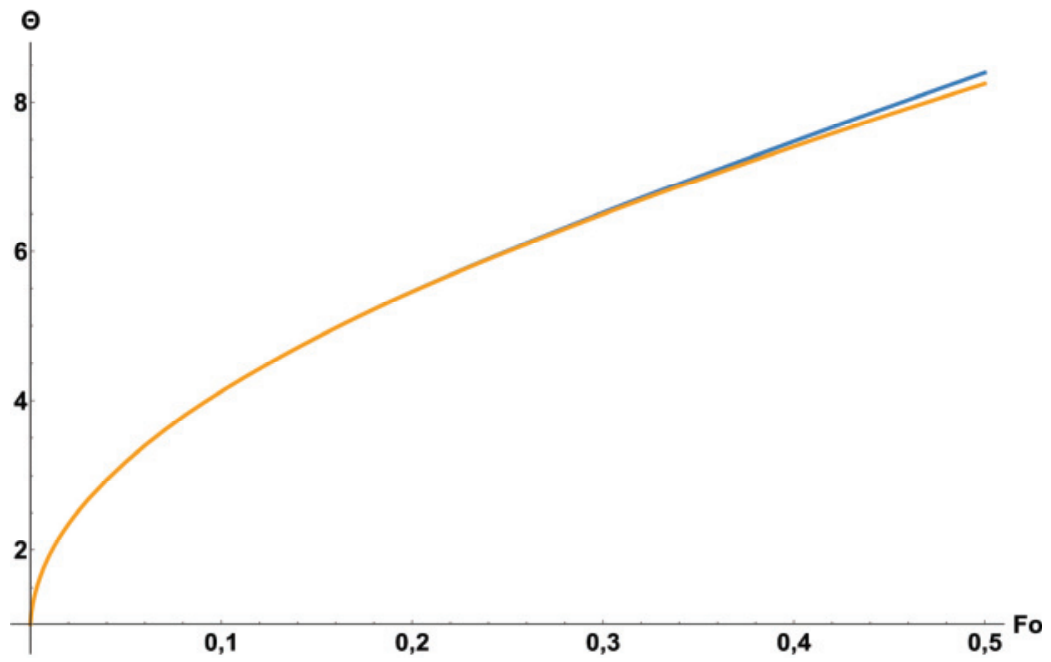

Pис. 7. График зависимости температур при разных числах Фурье: синяя линия-решение Лыкова [19], оранжевая-форлула 1.33

Fig. 7. Graph of temperature dependence for different Fourier numbers: blue line - Lykov [19] solution, orange - formula 1.33 


\section{Асимптотика при больших числах Fo (малые s)}

С этой целью разложим передаточную функцию в ряд при малых значениях оператора преобразования Лапласа $s$.

$F(X, s) \approx \varphi_{0}(X, s)+s \varphi_{1}(X, s)+s^{2} \varphi_{2}(X, s)+\ldots 1.35$

Подставляя 1.26 с учетом 1.35 в основное уравнение и приравнивая сомножители при одинаковых степенях $s$, получим зацепляющуюся систему уравнений второго порядка

$$
\left.\begin{array}{c}
\varphi_{0}^{\prime \prime}(X, s)=0 \\
\varphi_{1}^{\prime \prime}(X, s)=\varphi_{0}(X, s) \\
\varphi_{2}^{\prime \prime}(X, s)=\varphi_{1}(X, s)
\end{array}\right\} .
$$

Для решения системы уравнений 1.36 требуется каждому из них два граничных условия. Первое условие вытекает из 1.25

$$
\varphi_{0}^{\prime}(0, s)=0 ; \quad \varphi_{1}^{\prime}(0, s)=0 ; \quad \varphi_{2}^{\prime}(0, s)=0 .
$$

Вторая константа находится из интегральных соотношений

$$
\begin{gathered}
\int_{0}^{1} s \varphi_{0}(X, s) d x=1, \int_{0}^{1} \varphi_{1}(X, s) d X=\int_{0}^{1} \varphi_{0}(X, s) d X, \\
\int_{0}^{1} \varphi_{2}(X, s) d X=\int_{0}^{1} \varphi_{1}(X, s) d X=0 .
\end{gathered}
$$

Решая систему 1.36 с учетом граничных условий $1.37,1.38$, подставляя найденные сомножители $\varphi_{0}, \varphi_{1}, \varphi_{2} \ldots 1.35$ в 1.26 , выразим асимптотику решения при оставлении двух членов разложения 1.39:

$$
F(X, s) \simeq \varphi_{0}(X, s)+s \varphi_{1}(X, s) .
$$

В итоге после перехода от изображений к оригиналам получим выражение для температурного распределения 1.40:

$$
\begin{gathered}
\Theta(X, F O) \approx \\
\approx \Theta_{0}+\int_{0}^{F o}\left\{\begin{array}{l}
\left.K i_{w}-B i\left[\Theta_{w}(\eta)-1\right)\right]- \\
-S k\left[\Theta_{w}^{4}(\eta)-1\right]
\end{array}\right\} d \eta+ \\
+\left[K i-B i\left(\Theta_{w}-1\right)-S k\left(\Theta_{w}^{4}-1\right)\right] \frac{1-3 X^{2}}{6}+\ldots
\end{gathered}
$$

Неизвестная температура поверхности $\Theta_{w}$ найдется из интегрального уравнения Вольтерра II рода при $X=1$

$$
\begin{aligned}
& \Theta_{w} \approx \Theta_{0}+\int_{0}^{F o}\left\{\begin{array}{l}
\left.K i_{w}(\eta)-B i\left[\Theta_{w}(\eta)-1\right)\right]- \\
=S k\left[\Theta_{w}^{4}(\eta)-1\right]
\end{array}\right\}- \\
& -\frac{1}{3}\left[K i(F o)-B i\left(\Theta_{w}-1\right)-S k\left(\Theta_{w}^{4}-1\right)\right]+\ldots
\end{aligned}
$$

Решением 1.41 является интеграл

$$
\begin{aligned}
& 3\left(\mathrm{Fo}-\mathrm{FO}_{*}\right)=
\end{aligned}
$$

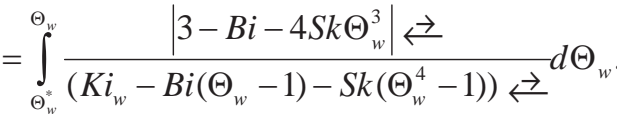

Здесь в первом приближении $\mathrm{Fo}^{*}$ и $\Theta_{w}^{*}$ - безразмерное время окончания асимптотического решения при малых Fо и температура поверхности в этот момент соответственно.

Раскрывая интеграл в 1.42 , можно в явном виде записать окончательное решение 1.43 для $\Theta_{w}$ при больших числах Fo

$$
3\left(\mathrm{Fo}-\mathrm{Fo}_{*}\right)=\Phi\left(\Theta_{w}\right)-\Phi\left(\Theta_{w}^{*}\right),
$$

где $\Phi(x)$ имеет вид

$$
\Phi(x)=\sum_{i=1}^{n} \frac{\left(\begin{array}{l}
\left.-3 \log \left[x-x_{i}\right]+B i \cdot \log \left[x-x_{i}\right]+\right) \\
+4 S k \log \left[x-x_{i}\right] x_{i}^{3}
\end{array}\right)}{B i+4 S k \cdot x_{i}^{3}},
$$

$x_{i}-$ корни уравнения

$$
K i_{w}+B i+S k-B i \cdot x_{i}-S k \cdot x_{i}^{4}=0 .
$$

Для записи корней выражения $1.44 x_{i}$ в общем виде введём следующие обозначения:

$$
\begin{aligned}
& 4\left(\frac{2}{3}\right)^{\frac{1}{3}}\left(K i_{w}+B i+S k\right)
\end{aligned}
$$

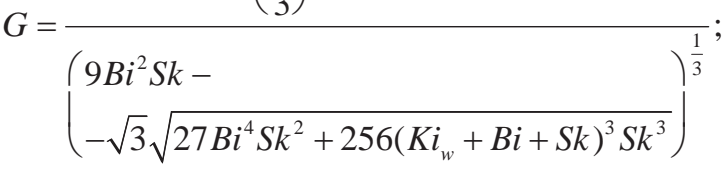

$$
\begin{aligned}
& H=\frac{\left(\begin{array}{l}
9 B i^{2} S k- \\
-\sqrt{3} \sqrt{27 B i^{4} S k^{2}+256\left(K i_{w}+B i+S k\right)^{3} S k^{3}}
\end{array}\right)^{\frac{1}{3}}}{2^{\frac{1}{3}} \cdot 3^{\frac{2}{3}} S k} ; \\
& I=\frac{2 B i}{S k \sqrt{-G+H}} \text {. }
\end{aligned}
$$

В итоге

$$
\begin{aligned}
& x_{1}=\frac{1}{2} \sqrt{-G+H}+\frac{1}{2} \sqrt{G-H-I} ; \\
& x_{2}=\frac{1}{2} \sqrt{-G+H}-\frac{1}{2} \sqrt{G-H-I} ; \\
& x_{3}=-\frac{1}{2} \sqrt{-G+H}-\frac{1}{2} \sqrt{G-H+I} ; \\
& x_{4}=-\frac{1}{2} \sqrt{-G+H}+\frac{1}{2} \sqrt{G-H+I} .
\end{aligned}
$$

По известной температуре поверхности может быть рассчитано и все температурное поле влажной пластины при больших Fo

$$
\begin{gathered}
\Theta(X, F O)=\Theta_{w}(F o)- \\
=\left[K i_{w}-B i\left(\Theta_{w}-1\right)-S k\left(\Theta_{w}^{4}-1\right)\right] \frac{1-3 X^{2}}{6}+\ldots
\end{gathered}
$$

Ниже приведено сравнение полученного асимптотического решения с расчетной задачей А.В. Лыкова [19] (рис. 8).

Начиная с Fo=0,3 погрешность решения составляет не более $1,4 \%$.

Отметим, что исследование теплопереноса проведено более детально, что и было реализовано при 


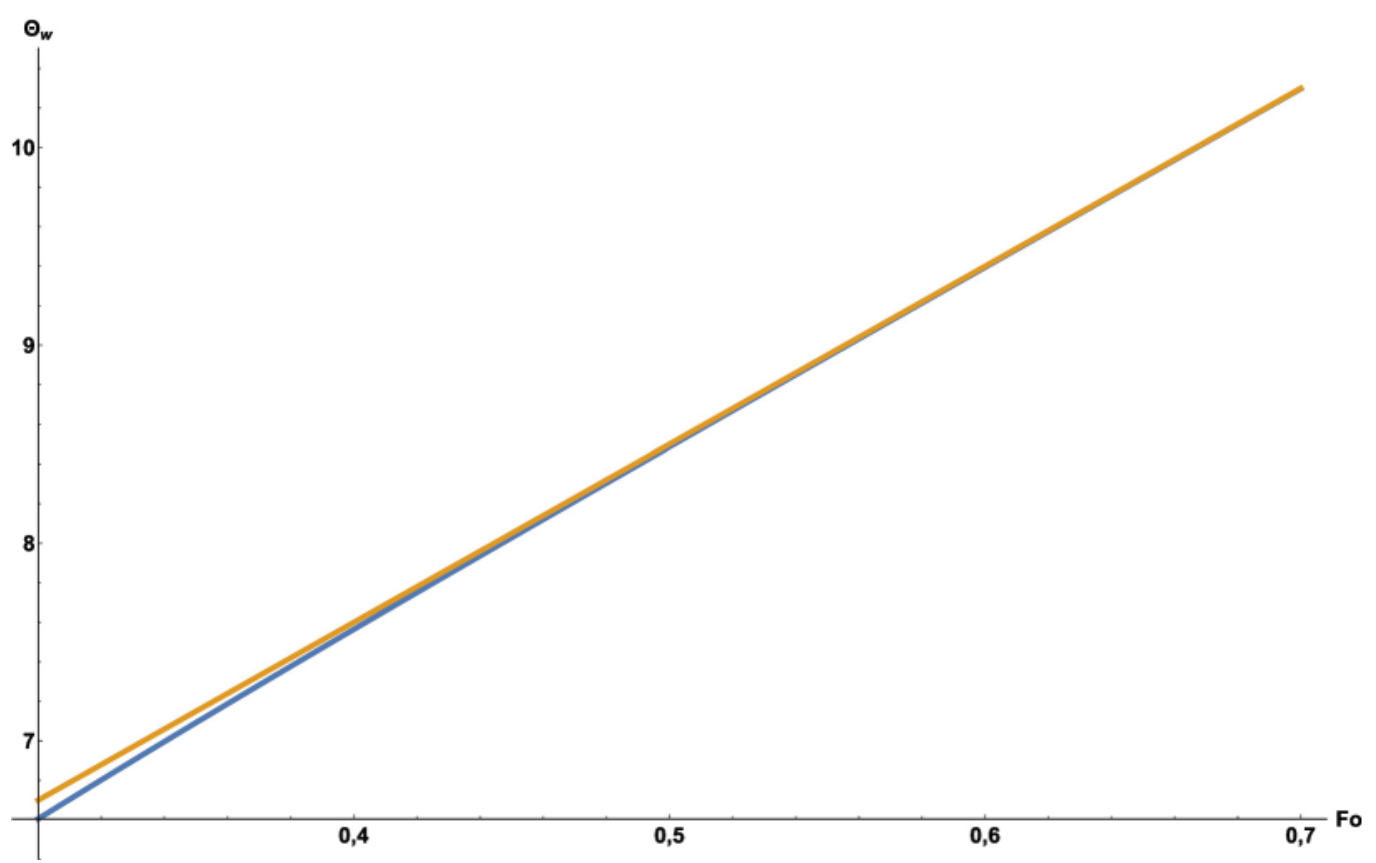

Рис. 8. Сравнение решений. Оранжевая линия - решение, полученное асимптотическим методом, синяя - решение Лыкова

Fig. 8. Comparison of solutions. Orange line is the solution obtained by the asymptotic method, blue is the Lykov solution

построении асимптотик. Для влагопереноса использовались опытно-корреляционные зависимости, полученные А.В. Лыковым [20]:

Скорость сушки в I периоде - периоде прогрева - может быть найдена из следующей зависимости 1.46 А.В. Лыкова [20]:

$$
\frac{d w}{d t_{I}}=\frac{d w}{d t} \cdot \frac{T(l, t)-T_{0}}{T_{M}-T_{0}} .
$$

Стыковка между первой и второй стадиями строится на следующем принципе. Так как температура поверхности влажной пластины в конце первого периода может быть рассчитана по соотношению 1.45 , это даёт возможность определить момент перехода стадии прогрева I в стадию II.

$$
T(l, t)=T_{y},
$$

$T(l, t)$ - установившаяся температура 1.47 поверхности влажного материала.

\section{Заключение}

Рассмотрен теоретический подход к решению начальной стадии прогрева влажного материала в нелинейной постановке. Метод основан на применении асимптотических процедур. Для малых значений безразмерного времени, что соответствует в пространстве изображений по Лапласу большим значениям параметра преобразования, асимптоти- ческое разложение строится в виде убывающего ряда по экспонентам, коэффициенты которого находятся из условия строгого удовлетворения основному уравнению второго порядка, две константы интегрирования которого определены: первая константа из условия симметрии, вторая - из интегрального условия. В результате получено приближенно-аналитическое решение существенно нелинейной задачи с радиационно-конвективным теплосбросом поглощенной СВЧ-энергии. Для больших значений безразмерного времени, что соответствует малым значениям параметра преобразования Лапласа, решение представляется в виде разложения по малому параметру, коэффициенты разложения которого находится аналогично первому случаю. В статье получено приближенно-аналитическое решение, учитывающего нелинейный характер теплового взаимодействия поверхности влажного массива с окружающей средой, с погрешностью расчетов не более $1,4 \%$ при оставлении только двух членов асимптотического разложения. Параметры влагопереноса определены в статье менее подробно, с использованием опытнокорреляционных зависимостей А.В. Лыкова.

Работа выполнена в ралках государственного задания ИТ СО РАН, при поддержке Российского фонда фундаментальных исследований, грант № 17-08-00752. 


\section{Список условных обозначений величин}

$W$ - плотность потока СВЧ-излучения, $\frac{\mathrm{BT}}{\mathrm{M}^{2}}$;

$A$ - коэффициент поглощения СВЧ-излучения ;

$q_{\text {w }}$ - плотность теплового потока;

$T$ - температура, $\kappa$;

и - влагосодержание;

$t$-время, с;

х - декартова координата, м;

l- полутолщина плоского слоя, м;

$c, \rho_{0}, \lambda, r, \varepsilon, a_{m}, a_{M}^{T}$ - теплофизические характеристики материала:

теплоемкость, плотность, теплопроводность, удельная теплота парообразования, коэффициенты испарения, диффузии влаги и терм одиффузии влаги соответственно; $\alpha$-коэффициент конвективного теплообмена, $\frac{\mathrm{B} т}{\mathrm{M}^{2} \mathrm{~K}}$;

$\varepsilon_{\text {ч }}$ - степень черноты поверхности;

$\sigma_{0}-$ константа теплового излучения абсолютно черн ой поверхности, $\frac{\mathrm{BT}}{\mathrm{M}^{2} \mathrm{~K}^{4}}$;

$T_{c}$ - температура окружающего пространства, $\mathrm{K}$;

$j$-плотность потока массы, $\frac{\kappa \Gamma}{\mathrm{M}^{2} \mathrm{c}}$;

$X=\frac{X}{l}-$ безразмерная координата;

Fo $=\frac{a t}{l^{2}}-$ число Фурье;

$L u=\frac{a_{m}}{a}-$ число Лыкова;

Ко $=\frac{r \overline{u_{0}}}{c T_{c}}-$ число Коссовича;

$P n=\frac{\delta \Delta T}{\Delta u}-$ число Поснова;

$P b=\frac{c b}{r}-$ число Ребиндера;

$\Theta(X, \mathrm{Fo})=\frac{T(x, t)}{T_{c}}-$ безразмерная температура;

$K i_{m}=\frac{j w l}{a_{m} \rho_{0} \overline{u_{0}}}-$ массобменный критерий Кирпичева;

$K i_{q}=\frac{q_{w} l}{\lambda T_{c}}-$ теплообменный критерий Кирпичева. 


\section{СПИСОК ЛИТЕРАТУРЫ}

1. Коновалов Н.П. Технология деструкции бурых углей методом нагрева энергией сверхвысоких частот. - Иркутск: Изд-во ИрГТУ, 2000. - 92 c.

2. Three-dimensional simulation of microwave heating coal sample with varying parameters / Yi-du Hong, Bai-quan Lin, He Li, Huaming Dai, Chuan-jie Zhu, HaoYao // Applied Thermal Engineering. - 25 January 2016. - V. 93. - P. 1145-1154

3. Sensitivity analysis on the microwave heating of coal: A coupled electromagnetic and heat transfer model / Baiquan Lin, He Li, Zhongwei Chen, Chunshan Zheng, Yidu Hong, ZhengWang // Applied Thermal Engineering. - 5 November 2017. - V. 126. P. 949-962.

4. Meda V., Orsat V., Raghavan V. Microwave heating and the dielectric properties of foods / Woodhead Publishing Series in Food Science, Technology and Nutrition. - Cambridge: Woodhead Publ., 2017. - P. 23-43.

5. Microwave drying performance of single-particle coal slime and energy consumption analyses / Zhanlong Song, Chuanming Jing, Liansheng Yao, Xiqiang Zhao, Wenlong Wang, Yanpeng Mao, Chunyuan Ma // Fuel Processing Technology. - March 2016. V. 143. - P. 69-78.

6. Faisal Mushtaq, Ramli Mat, Farid Nasir Ani. Fuel production from microwave assisted pyrolysis of coal with carbon surfaces // Energy Conversion and Management. - 15 February 2016. V. $110 .-$ P. $142-153$.

7. Drying behavior of lignite under microwave heating / Zhanlong Song, Liansheng Yao, Chuanming Jing, Xiqiang Zhao, Wenlong Wang \& Chunyuan Ma // Drying Technology. - 31 Jan 2017. V. 35. - Iss. 4. - P. 433-443.

8. Radha Raman Mishra, Apurbba Kumar Sharma Microwave-material interaction phenomena: Heating mechanisms, challenges and opportunities in material processing // Composites Part A: Applied Science and Manufacturing. - February 2016. - V. 81. P. 78-97.

9. Temperature rise characteristics of Zhun Dong coal during microwave pyrolysis / Huan-Peng Liu, Tai-Ping Chen, Yang Li, Zhen-Yu Song, Shao-Wei Wang, Shao-Hua Wu / / Fuel Processing Technology. - July 2016. - V. 148. - P. 317-323.

10. Хайдурова А.А., Федчишин В.В., Коновалов Н.П. Микроволновая сушка бурых углей и повышение их технологических характеристик // Изв. ВУЗов. Проблемы энергетики. - Казань: Изд-во КГЭУ, 2010. - С. 31-35.
11. Methods and systems for processing solid fuel. US20090272028A1 United States.

12. Effect of microwave irradiation on the preparation of iron oxide/arenaceous clay sorbent for hot coal gas desulfurization / Yu Feng, Tianqi Hu, Mengmeng Wu, Ju Shangguan, Huilin Fan, Jie Mi // Fuel Processing Technology. - July 2016. - V. 148. P. 35-42.

13. Experimental study on the petrophysical variation of different rank coals with microwave treatment / $\mathrm{He} \mathrm{Li}$, Baiquan Lin, Wei Yang, Chunshan Zheng, Yidu Hong, Yabin Gao, Tong Liu, Shiliang Wu // International Journal of Coal Geology. - 15 January 2016. - V. 154-155. - P. 82-91.

14. Activated carbon obtained from sapelli wood sawdust by microwave heating for 0-cresol adsorption / P.S. Thue, G.S. dos Reis, E.C. Lima, J.M. Sieliechi, G.L. Dotto, A.G.N. Wamba, S.L.P. Dias, F.A. Pavan // Research on Chemical Intermediates. - February 2017. - V. 43. - Iss. 2. - P. 1063-1087.

15. Effect of microwave irradiation on petrophysical characterization of coals / Hong Yi-du, Lin Bai-quan, Zhu Chuan-jie, Li He // Applied Thermal Engineering. - 5 June 2016. - V. 102. P. 1109-1125.

16. Jing Sun, Wenlong Wang, Qinyan Yue. Review on MicrowaveMatter Interaction Fundamentals and Efficient Microwave-Associated Heating Strategies // Materials. - 2016. - P. 231. DOI: $10.3390 / \mathrm{ma} 9040231$

17. Rajasekhar Reddy B., Vinu R. Microwave assisted pyrolysis of Indian and Indonesian coals and product characterization // Fuel Processing Technology. - 15 December 2016. - V. 154. P. 96-103.

18. Microwave absorption characteristics of anthracite during pyrolysis / Zhiwei Peng, Xiaolong Lin, Xuejiao Wu, Jiann-Yang Hwang, Byoung-Gon Kim, Yuanbo Zhang, Guanghui Li, Tao Jiang // Fuel Processing Technology. - September 2016. V. 150 . - P. 58-63.

19. Лыков А.В. Теория теплопроводности. - М.: Изд-во «Высшая школа» 1967. - 600 с.

20. Лыков А.В. Теория сушки. - М.: Изд-во «Энергия», 1968. $471 \mathrm{c.}$

Поступила 21.01.2019 2.

\section{Информация об авторах}

Саломатов B.B., доктор технических наук, профессор кафедры физики неравновесных процессов Новосибирского государственного университета; главный научный сотрудник Института теплофизики им. С.С. Кутателадзе СО РАН.

Kарелин B.A., аспирант кафедры физики неравновесных процессов Новосибирского государственного университета; инженер-исследователь Института теплофизики им. С.С. Кутателадзе СО РАН.

Саломатов B.B., кандидат физико-математических наук, ведущий программист института теплофизики им. С.С. Кутателадзе СО РАН. 
UDC 621.311

\title{
ELECTROMAGNETIC DRYING OF WET MATERIALS WITH MICROWAVE LOW PENETRATION DEPTH IN CONDITIONS OF HEAT REMOVAL BY RADIATION AND CONVECTION. I. WARM UP PHASE
}

\author{
Vladimir V. Salomatovi, \\ salomatov.vv@mail.ru \\ Vadim A. Karelin ${ }^{1,2}$ \\ vad2hen@mail.ru \\ Vasiliy V. Salomatov², \\ vvsalomatov@mail.ru \\ ${ }^{1}$ Novosibirsk State University, \\ 1, Pirogov street, Novosibirsk, 630090, Russia \\ 2 S.S. Kutateladze Institute of Thermophysics SB RAS, \\ 1, Akademician Lavrentiev avenue, Novosibirsk, 630090, Russia.
}

\begin{abstract}
The relevance of the research is determined by the need to develop mathematical models of microwave heating and MW-drying of wet materials to obtain technologically optimal and cost-effective modes. Due to the complexity and non-linearity of the processes taking place inside wet materials during microwave processing, it is extremely important to build models that would allow one to construct analytically approximate solutions in order to find the main laws and characteristic features of the processes under consideration.

The aim of the research is to state the first stage of the microwave drying of wet material - the warm-up phase. The search for analytical solution for the temperature of the layer at different points in time allows us to determine the beginning of the next stage - drying.

The object of the research is a flat layer of wet material - coal, sand, wood and other capillary-porous arrays, which are affected by microwave radiation. Such materials have a high dielectric constant and, as a result, they very efficiently absorb microwave radiation, which is almost $100 \%$ converted to thermal energy.

The methods are associated with mathematical modeling, which are based on Maxwell electrodynamics equations and heat and moisture transfer of A.V. Lykov. In this article, the Maxwell problem is solved independently on the problem of heat and mass transfer; therefore, the flux density of the absorbed microwave radiation is assumed to be known. Also, one of the features of this problem is the consideration of materials with a small depth of absorption, whereby the source term in the system of equations for heating is in the boundary condition.

As a result of the research the problem of heat transfer at a given power source of heat was solved using asymptotic procedures. The authors have obtained approximate analytical solutions for the first of the three characteristic stages of microwave drying under conditions of low penetration depth of microwaves: the warm-up stage of a moist material. The constructed solutions were analyzed.
\end{abstract}

Key words:

Microwave energy, drying, capillary-porous massif, heat radiation, convection, A.V. Lykov heat and moisture transfer equations.

The research was carried out within the government assignment for IT SB RAS with support of the Russian Foundation for Basic Research, grant no. 17-08-00752

\section{REFERENCES}

1. Konovalov N.P. Tekhnologiya destruktsii burykh ugley metodom nagreva energiey suerkhvysokikh chastot [Technology of brown coal destruction by the method of heating by energy of ultrahigh frequencies]. Irkutsk, IrSTU Publ. house, 2000. 92 p.

2. Yi-du Hong, Bai-quan Lin, He Li, Hua-ming Dai, Chuan-jie Zhu, HaoYao. Three-dimensional simulation of microwave heating coal sample with varying parameters. Applied Thermal Engineering, 25 January 2016, vol. 93, pp. 1145-1154.

3. Baiquan Lin, He Li, Zhongwei Chen, Chunshan Zheng, Yidu Hong, Zheng Wang. Sensitivity analysis on the microwave heating of coal: A coupled electromagnetic and heat transfer model. Applied Thermal Engineering, 5 November 2017, vol. 126, pp. 949-962.

4. Meda V., Orsat V., Raghavan V. Microwave heating and the dielectric properties of foods. Woodhead Publishing Series in Food Science, Technology and Nutrition. Cambridge, Woodhead Publ., 2017. pp. 23-43.

5. Zhanlong Song, Chuanming Jing, Liansheng Yao, Xiqiang Zhao, Wenlong Wang, Yanpeng Mao, Chunyuan Ma. Microwave drying performance of single-particle coal slime and energy consumption analyses. Fuel Processing Technology, March 2016, vol. 143, pp. $69-78$.

6. Faisal Mushtaq, Ramli Mat, Farid Nasir Ani. Fuel production from microwave assisted pyrolysis of coal with carbon surfaces. Energy Conversion and Management, 15 February 2016, vol. 110, pp. 142-153.

7. Zhanlong Song, Liansheng Yao, Chuanming Jing, Xiqiang Zhao, Wenlong Wang \& Chunyuan Ma. Drying behavior of lignite under microwave heating. Drying Technology, 31 Jan 2017, vol. 35, Iss. 4, pp. 433-443.

8. Radha Raman Mishra, Apurbba Kumar Sharma. Microwave-material interaction phenomena: Heating mechanisms, challenges and opportunities in material processing. Composites Part A: Applied Science and Manufacturing, February 2016, vol. 81, pp. 78-97.

9. Huan-Peng Liu, Tai-Ping Chen, Yang Li, Zhen-Yu Song, ShaoWei Wang, Shao-Hua Wu. Temperature rise characteristics of Zhun Dong coal during microwave pyrolysis. Fuel Processing Technology, July 2016, vol. 148, pp. 317-323. 
10. Khaidurova A.A., Fedchishin V.V., Konovalov N.P. Microwave drying of brown coals and increasing their technological characteristics. Izvestiya Universities. Problems of energy. Kazan, KGEU Publ. house, 2010. pp. 31-35. In Rus.

11. Drozd J.M., Druga M.C., Lawson S.L., Lang F.Ch., Bullis H.L. Methods and systems for processing solid fuel. US20090272028A1 United States.

12. Yu Feng, Tianqi Hu, Mengmeng Wu, Ju Shangguan, Huilin Fan, Jie Mi. Effect of microwave irradiation on the preparation of iron oxide/arenaceous clay sorbent for hot coal gas desulfurization. Fuel Processing Technology, July 2016, vol. 148, pp. 35-42.

13. He Li, Baiquan Lin, Wei Yang, Chunshan Zheng, Yidu Hong, Yabin Gao, Tong Liu, Shiliang Wu. Experimental study on the petrophysical variation of different rank coals with microwave treatment. International Journal of Coal Geology, 15 January 2016, vol. $154-155$, pp. 82-91.

14. Thue P.S., Dos Reis G.S., Lima E.C., Sieliechi J.M., Dotto G.L., Wamba A.G.N., Dias S.L.P., Pavan F.A. Activated carbon obtained from sapelli wood sawdust by microwave heating for 0 -cresol adsorption. Research on Chemical Intermediates, February 2017, vol. 43, Iss. 2, pp. 1063-1087.
15. Hong Yi-du, Lin Bai-quan, Zhu Chuan-jie, Li He. Effect of microwave irradiation on petrophysical characterization of coals. Applied Thermal Engineering, 5 June 2016, vol. 102, pp. 1109-1125.

16. Jing Sun, Wenlong Wang, Qinyan Yue. Review on MicrowaveMatter Interaction Fundamentals and Efficient Microwave-Associated Heating Strategies. Materials, 2016. pp. 231. DOI: $10.3390 / \mathrm{ma} 9040231$

17. Rajasekhar Reddy B., Vinu R. Microwave assisted pyrolysis of Indian and Indonesian coals and product characterization. Fuel Processing Technology, 15 December 2016, vol. 154, pp. 96-103.

18. Zhiwei Peng, Xiaolong Lin, Xuejiao Wu, Jiann-Yang Hwang, Byoung-Gon Kim, Yuanbo Zhang, Guanghui Li, Tao Jiang. Microwave absorption characteristics of anthracite during pyrolysis. Fuel Processing Technology, September 2016, vol. 150, pp. 58-63.

19. Lykov A.V. Teoriya teploprovodnosti [Theory of heat conductivity]. Moscow, Vysshaya shkola Publ., 1967. 600 p.

20. Lykov A.V. Teoriya sushki [The theory of drying]. Moscow, Energiya Publ., 1968. $471 \mathrm{p}$.

\section{Information about the authors}

Vladimir V. Salomatov, Dr. Sc., professor, Novosibirsk State University; leading researcher, S.S. Kutateladze Institute of Thermophysics SB RAS.

Vadim A. Karelin, postgraduate, Novosibirsk State University; engineer, S.S. Kutateladze Institute of Thermophysics SB RAS.

Vasiliy V. Salomatov, Cand. Sc., leading programmer S.S. Kutateladze Institute of Thermophysics SB RAS. 